\title{
Covert pharmaceutical promotion in free medical journals
}

\author{
Aaron S. Kesselheim MD JD
}

See related research article by Becker and colleagues, page 544

Competing interests: Aaron Kesselheim is a consultant for the Alosa Foundation, a nonprofit organization that is not affiliated with any pharmaceutical company. He has served as an expert witness for the State of Texas in a lawsuit against Merck concerning the improper promotion of rofecoxib. From 2007 to 2008, he helped develop an educational program to encourage evidence-based prescribing practices, funded by the Attorney General Consumer and Prescriber Education Grant Program.

This article was solicited and has not been peer reviewed.

Correspondence to: Dr. Aaron S. Kesselheim, akesselheim@partners.org

CMAJ 2011. DOI: 10.1503 /cmaj.110156
$\mathrm{T}$ he influence of the promotional practices of pharmaceutical manufacturers on the prescribing behaviour of physicians has long been recognized. Since the early 1980s, studies of this phenomenon have repeatedly shown that physicians' knowledge of drug properties is more consistent with sales information than with the evidence published in the medical literature, even though physicians generally deny such an influence. ${ }^{1}$

Over the past decade, the direct-to-physician promotion of pharmaceuticals has led to the widespread inappropriate use of drugs such as rofecoxib, rosiglitazone and certain antipsychotic drugs, ${ }^{2}$ which in turn has resulted in increased morbidity and mortality for patients. Commercial sources of information for these and other products have consistently overstated their benefits and underestimated their risks. In response, experts on health policy have called for better oversight of the interactions between physicians and the pharmaceutical industry, ${ }^{3}$ and some institutions have placed stringent limits on the interactions between physicians and marketing representatives. ${ }^{4}$

Efforts to stem the influence of marketing strategies on the prescribing behaviour of physicians may be limited because some strategies occur beyond the authority of regulators and public watchdogs. ${ }^{5}$ For example, insiders from one pharmaceutical manufacturer recently alleged that websites maintained by third parties but funded and controlled by the manufacturer promoted unapproved off-label uses of the product. ${ }^{6}$ Some physicians have pointed to nominally

\section{- KEY POINTS}

- The promotion of pharmaceuticals to physicians in free medical journals may escape regulation or oversight.

- Biased reviews of drugs and the advertising published in free medical journals may lead to overuse or inappropriate off-label use of drugs.

- The reliance on free medical journals for continuing medical education could be reduced by creating and disseminating consumer-friendly, evidence-based alternatives with government or other support not affiliated with industry. independent private organizations that hold continuing medical education (CME) conferences and have suggested that receiving funding from the pharmaceutical industry makes these organizations susceptible to the delivery of inappropriate promotional messages.?

In this issue of CMAJ, Becker and colleagues provide an in-depth look at another potential source of the covert promotion of pharmaceuticals: the free ("controlled-circulation") medical journal. ${ }^{8}$ The authors focus on publications from Germany, but there are versions of these journals in virtually every other major medical marketplace. In the United States, these publications may be sent to the membership lists of the American Medical Association (AMA) or a specialty society. Controlled-circulation publications are disseminated without subscription because they are funded entirely by advertising revenue. Many provide summaries of previously published peerreviewed research in easily read formats that may appeal to busy physicians. Some articles are specifically intended for CME purposes and may provide physicians with the opportunity to apply for the credits required by regulatory authorities that show their ability to absorb the information contained in the articles. For many physicians, obtaining CME credits in this manner may be less expensive and more convenient than attending a course or conference.

The autonomy of controlled-circulation publications has been a perennial subject of debate. In the 1970s, Richard Crout, head of the Bureau of Drugs at the US Food and Drug Administration, testified before Congress that there was "evidence of ghostwritten articles and heavily biased therapeutic reviews in controlled circulation journals that included quizzes at the end that could be mailed in to receive AMA-accredited CME credits."

When the editors of peer-reviewed journals discuss their policies on advertising, free publications act as examples of what not to do by showing how editorial independence might be lost. ${ }^{10,11}$ Earlier this year, a well-known psychiatrist posted links to a free publication in which supplements for $\mathrm{CME}$

All editorial matter in CMAJ represents the opinions of the authors and not necessarily those of the Canadian Medical Association. 
may have unduly favoured or omitted important safety information about the drugs sold by the manufacturers who sponsored the publication. ${ }^{12}$

Becker and colleagues provide valuable empirical data to inform the discussion over the role these publications play in medical education. ${ }^{8}$ In a cohort of 11 German periodicals, the authors systematically examined pharmaceutical advertisements and CME articles discussing certain heavily marketed drugs or drug classes. ${ }^{8}$ Five of these publications were distributed to physicians for free (i.e., the publications were supported entirely by advertising revenue), one relied on a revenue model that combined subscription fees and paid advertisements, and five journals were available by subscription only and included no advertisements. ${ }^{8}$ The authors found a greater number of articles that mentioned the selected drugs in the free publications than in the subscription publications. ${ }^{8}$ The authors also found that nearly all of the articles in the free publications depicted the drugs in a positive light. In two of the free publications, logistic regression analysis helped the authors to conclude that the presence of an advertisement for a specific drug more than doubled the odds of an article appearing in the same issue that made a positive recommendation for the use of the drug. ${ }^{8}$

The data do not allow us to determine whether this bias is conscious or subconscious, but the results reported by Becker and colleagues are nonetheless salient. Covert promotion of pharmaceuticals is an important public health issue because it can contribute to the unnecessary overuse of certain drugs or lead to their off-label use without sufficient evidence of efficacy.

Addressing the problem may be difficult. In most jurisdictions, these journals cannot be banned, and regulators have little authority to oversee editorial decisions. However, their impact may be lessened if medical societies refused to be complicit and stopped selling their membership rosters, or if professional organizations such as the US Accreditation Council for Continuing Medical Education did not certify CME credits sought by physicians for reading these publications.

Becker and colleagues suggest addressing the public health issue by encouraging physicians to rely mainly on journals supported by subscription fees for their CME. ${ }^{8}$ This suggestion is reminiscent of that of Rennie and Bero, who proposed in 1990 that physicians return free publications, at cost, to the publishers. ${ }^{13}$ Twenty years later, however, free publications are still prominent. Reducing their influence will require increasing the awareness among physicians of the bias that can be contained in the pages of these publications. It will also require competition from high-quality alternatives that meet the physicians' demand for readable summaries of recent medical literature, as well as updates on evidence-based prescribing and the management of certain clinical conditions. Further funding from governments or other sources not affiliated with the pharmaceutical industry for the development of such information would promote rational prescribing and better outcomes while reducing the need for prescription fees. Most physicians would be happy to receive additional information about the proper use of drugs that is both effective and free of bias, but the medical community needs to do a better job of creating and disseminating this information without the influence of pharmaceutical advertising.

\section{References}

1. Avorn J, Chen M, Hartley R. Scientific versus commercial sources of influence on the prescribing behavior of physicians. Am J Med 1982;73:4-8.

2. Alexander GC, Gallagher SA, Mascola A, Moloney RM, Stafford RS. Increasing off-label use of antipsychotic medications in the United States, 1995-2008. Pharmacoepidemiol Drug Saf 2011; 20:177-84.

3. Brennan TA, Rothman DJ, Blank L, et al. Health industry practices that create conflicts of interest: a policy proposal for academic medical centers. JAMA 2006;295:429-33.

4. Pollack A. Stanford to ban drug makers' gifts to doctors, even pens. New York Times 2006 Sept. 12. Available: www.nytimes .com/2006/09/12/business/12drug.html (accessed 2011 Feb. 8)

5. Mello MM, Studdert DM, Brennan TA. Shifting terrain in the regulation of off-label promotion of pharmaceuticals. N Engl J Med 2009;360:1557-66.

6. United States ex rel. Lang and Rushin v. Allergan, Civ. No. 1:07-CV-01288-WSD (N.D. ga.) (filed 2007 June 5).

7. Singer N, Wilson D. Debate over industry role in educating doctors. New York Times 2010 June 23. Available: www.nytimes.com /2010/06/24/business/24meded.html?_r=1 (accessed 2011 Feb. 8)

8. Becker A, Dörter F, Eckhardt K, et al. The association between a journal's source of revenue and the drug recommendations made in the articles it publishes. CMAJ 2011;183:544-8.

9. Greene JA, Podolsky SH. Keeping modern in medicine: pharmaceutical promotion and physician education in postwar America. Bull Hist Med 2009;83:331-77.

10. McDonnell PJ. Let the buyer (and reader) beware!: targeted advertising in medical journals. Arch Ophthalmol 1999;117:1077.

11. Smith R. Should medical journals carry drug advertising? $B M J$ 2007;335:74

12. Carlat D. "Current psychiatry": now binging on industry cash for CME [blog]. 2011 Jan. 4. Available: http://carlatpsychiatry .blogspot.com/2011/01/current-psychiatry-now-binging-on.html (accessed 2011 Jan. 24)

13. Rennie D, Bero LA. Throw it away, Sam: the controlled circulation journals. AJR Am J Roentgenol 1990;155:889-92.

Affiliation: Aaron Kesselheim is with the Division of Pharmacoepidemiology and Pharmacoeconomics, Department of Medicine, Brigham and Women's Hospital and Harvard Medical School, Boston, Mass.

Funding: Aaron Kesselheim is supported by a career development award from the Agency for Healthcare Research and Quality (grant no. K08HS18465-01) and a Robert Wood Johnson Foundation Investigator Award in Health Policy Research. 